\title{
Innovación docente en Biomedicina. Un recorrido de acercamiento a práctica clínica habitual. La investigación y su inclusión en una consulta médica.
}

\section{Teaching innovation in Biomedicine. A journey of approach to regular clinical practice. Research and its inclusion in a medical practice.}

ALEJANDRO MUÑOZ JIMÉNEZ

https://orcid.org/0000-0001-8884-9225

Universidad de Sevilla

Departamento de Medicina

amunoz23@us.es.

DOI: http://dx.doi.org/10.12795/9788447231003.051

Pp.: 1067-1087 


\section{Descripción del contexto}

La asignatura en la que se desarrolla el Ciclo de Mejora en el Aula (CIMA) forma parte de las prácticas en "aula" de la asignatura Patología General del Grado de Biomedicina, a lo largo de su segundo curso.

La docencia impartida se realiza a través de seminarios-taller que pretenden acercar al alumno a situaciones clínicas reales, vividas en el ámbito de una consulta médica o planta de hospitalización. Dada la situación de Pandemia por COVID19, las prácticas dentro del hospital y/o consultas externas han sido suspendidas. De este modo, se realiza un programa de clases prácticas desarrolladas en aula, para acercar al alumnado a la práctica clínica habitual. Son clases de 6 alumnos, que se desarrollan en horario de 9:00-13:00h en las aulas del pabellón Docente del H. U. Virgen del Rocío (Sevilla).

\section{Diseño del CIMA}

\section{Mapa de contenidos y problemas}

Los contenidos de este CIMA se distribuyen según el esquema de contenidos estructurales y no estructurales, conceptuales, de datos, procedimentales (psicomotriz e intelectual) y aquellos que promueven la actitud del alumno (Figura 1).

Ciclos de Mejora en el Aula (2020). Experiencias de Innovación Docente de la US Esta obra se distribuye con la licencia Creative Commons 


\section{Sarcopenia y COVID19}

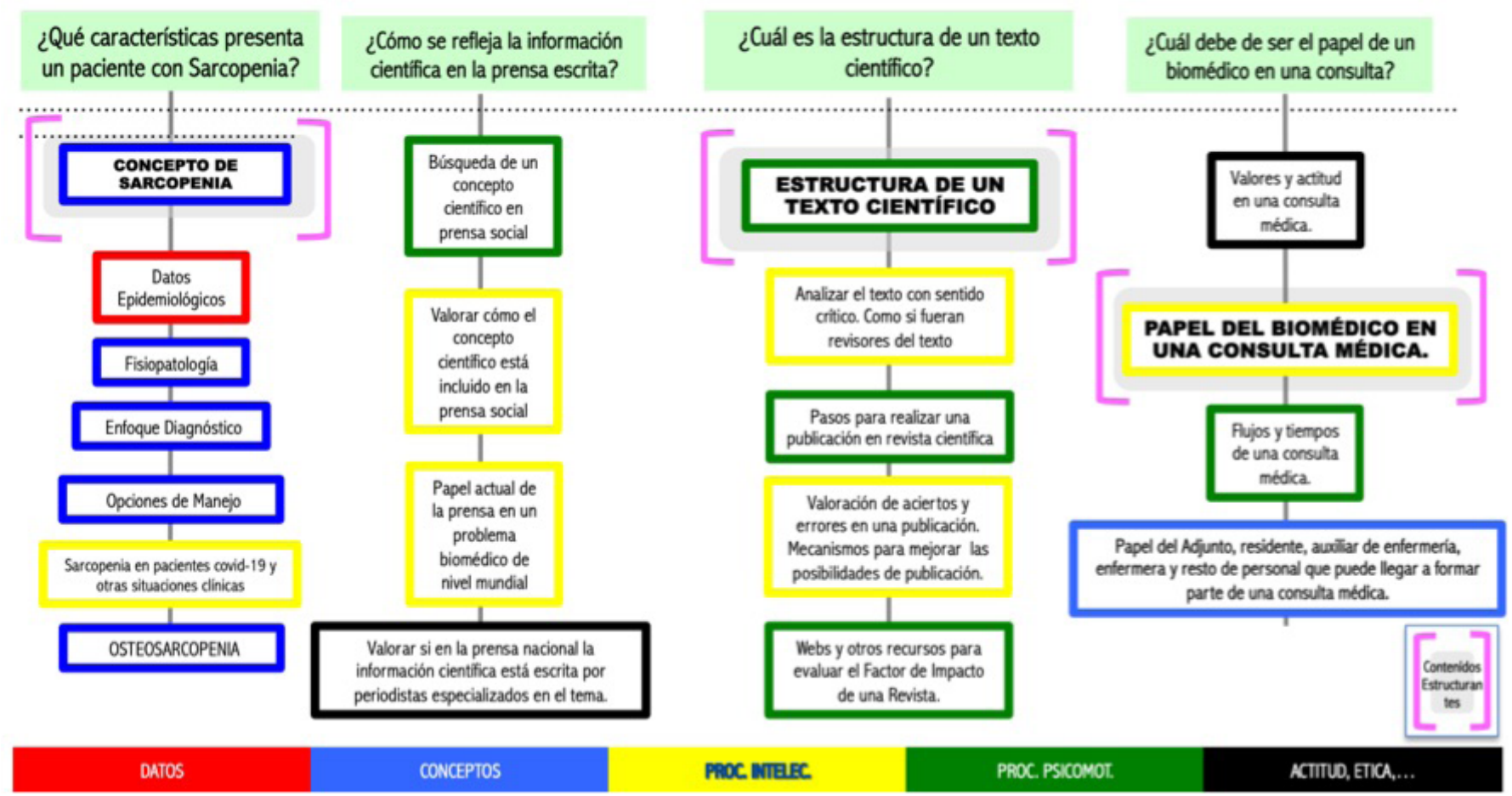

Figura 1. Mapa de contenidos y problemas

Ciclos de Mejora en el Aula (2020). Experiencias de Innovación Docente de la US

(c) (i) $\odot$ Esta obra se distribuye con la licencia Creative Commons 
Este CIMA desarrolla el concepto de sarcopenia. A raíz de este concepto, se desarrollan 4 grandes bloques que intentan acercar al alumno a la práctica clínica habitual de una consulta médica. El concepto en sí es desconocido por los alumnos, de forma específica. Gracias al wooclap se permite conocer la zona de desarrollo próxima (ZDP) del grupo (Derouesn, 2017). El enfoque de este concepto en la prensa actual y su relación a la infección por COVID-19 pretende gestionar en el alumnado una búsqueda activa de la información en la prensa actual. De un texto social o civil, pasamos a un texto científico, relacionado con la sarcopenia y la infección por COVID-19, para realizar un ejercicio activo de poder revisar la estructura y el contenido de un texto científico. Se finaliza el proceso con un "role play" destinado, como no podía ser de otro modo, a "imitar" aquello que ocurre en el interior de una consulta médica, tras la valoración de un paciente infectado por COVID-19, que tras un pregunta problema cambia su estatus clínico. En este apartado se pretende valorar el papel actual y futuro del biomédico en un contexto eminentemente clínico, como es la consulta médica.

\section{Modelo metodológico posible}

El aprendizaje que se pretende alcanzar se produce cuando los alumnos desarrollan las competencias básicas y son capaces de aprender a aprender. Para ello se desarrolla un modelo metodológico posible (Figura 2) que está enfocado a la consecución de un aprendizaje significativo, donde la adquisición de conocimientos tiene sentido para el estudiante y este puede aplicar lo aprendido a su vida cotidiana.

Ciclos de Mejora en el Aula (2020). Experiencias de Innovación Docente de la US Esta obra se distribuye con la licencia Creative Commons 


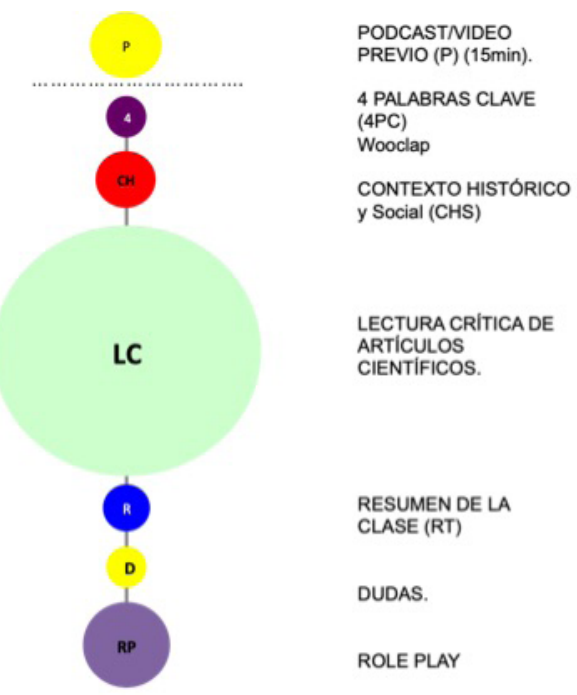

Figura 2. Modelo metodológico posible

\section{Secuencia de actividades programadas}

Las actividades programadas se establecen según una secuencia, que intenta acercar al alumno a la práctica clínica habitual de una consulta médica. Lo primero es que unas 24-48 horas antes de la clase se le envía un video o podcast explicando de forma didáctica los datos y conceptos más importantes acerca de la sarcopenia y su relación actual con la infección por COVID-19. Se trata de un video de unos 15 minutos que permiten iniciar la clase de una forma más dinámica. Gracias al wooclap se permite conocer la zona de desarrollo próxima (ZDP) del grupo.? El enfoque de este concepto en la prensa actual y su relación a la infección por COVID-19 pretende gestionar en el alumnado una búsqueda activa de la información en la prensa actual. Una vez completado este paso, la clase se dirige a analizar de forma activa la estructura y el contenido de un texto científico (Tabla 2). Se finaliza la clase con un resumen, resolución de dudas y finalmente con el desarrollo de un "role play" con el contexto siguiente: paciente infectado por COVID-19 y su flujo dentro de una consulta protagonizada por médicos y biomédicos (Tabla 3).

Ciclos de Mejora en el Aula (2020). Experiencias de Innovación Docente de la US Esta obra se distribuye con la licencia Creative Commons 
Tabla 1. Secuencias de actividades programadas. P1 (PodCast/video previo), 4 (4 palabras claves con el uso de wooclap). CH (Contexto histórico)

\begin{tabular}{|c|c|c|c|c|c|}
\hline Actividad & Descripción & Contenido & Materiales & Finalidad & Tiempo \\
\hline P1 & $\begin{array}{c}\text { Podcast/ } \\
\text { video Previo }\end{array}$ & $\begin{array}{l}15 \text { min de clase teórica que se } \\
\text { enviará } 48 \text { antes de la clase. }\end{array}$ & ScreenCast & $\begin{array}{l}\text { Llegar a la clase con un } \\
\text { conocimiento teórico previo } \\
\text { ya adquirido }\end{array}$ & $15^{\prime}$ \\
\hline 4 & $\begin{array}{l}4 \text { Palabras } \\
\text { Clave }\end{array}$ & $\begin{array}{l}\text { Se solicitará al grupo que envien } \\
4 \text { palabras claves sobre el } \\
\text { concepto tratado en la clase } \\
\text { ("SARCOPENIACOVID 19") }\end{array}$ & Wooclap & $\begin{array}{l}\text { Conocer el ZDP (zona de } \\
\text { desarrollo próximo) del grupo } \\
\text { tras haber escuchado el } \\
\text { vídeo previo }\end{array}$ & $10^{\prime}$ \\
\hline $\mathrm{CH}$ & $\begin{array}{l}\text { Contexto } \\
\text { Histórico y } \\
\text { Social }\end{array}$ & $\begin{array}{l}\text { Coloquio sobre el contexto } \\
\text { histórico y social del tema a } \\
\text { tratar }\end{array}$ & $\begin{array}{l}\text { Prensa } \\
\text { nacional }\end{array}$ & $\begin{array}{l}\text { Contextualizar en el } \\
\text { momento actual y pasado el } \\
\text { problema a tratar } \\
\text { (antecedentes y contexto } \\
\text { actual del tema) }\end{array}$ & $15^{\prime}$ \\
\hline
\end{tabular}

Tabla 2. Secuencias de actividades programadas. LC (Lectura crítica)

\begin{tabular}{|c|c|c|c|c|c|}
\hline Actividad & Descripción & Contenido & Materiales & Finalidad & Tiempo \\
\hline LC & $\begin{array}{l}\text { Lectura Crítica } \\
\text { de artículos } \\
\text { científicos } \\
\text { relacionados } \\
\text { con el tema }\end{array}$ & $\begin{array}{l}\text { Artículos que por su alto factor de } \\
\text { impacto o por su originalidad, } \\
\text { aportarán una perspectiva } \\
\text { integradora del contenido teórico } \\
\text { expresado. } \\
\text { Se iniciará un subrayado sobre el } \\
\text { papel (en grupo) y tras el mismo } \\
\text { el profesor realizará preguntas } \\
\text { que intentarán conocer no sólo el }\end{array}$ & $\begin{array}{l}\text { 2-3 artículos con } \\
\text { relevancia } \\
\text { científica. }\end{array}$ & $\begin{array}{l}\text { Gracias al contexto } \\
\text { que ofrece el } \\
\text { artículo cientifico, } \\
\text { nos permitirá } \\
\text { trabajar sobre los } \\
\text { conceptos de: } \\
\text { diagnóstico, } \\
\text { tratamiento y } \\
\text { pronóstico. }\end{array}$ & $120^{\prime}$ \\
\hline & & $\begin{array}{l}\text { contenido científico, sino también } \\
\text { la estructura del artículo. }\end{array}$ & & & \\
\hline
\end{tabular}

Tabla 3. Secuencias de actividades programadas. R (Resumen de la clase), D (Dudas). RP (role play)

\begin{tabular}{|c|c|c|c|c|c|}
\hline Actividad & Descripción & Contenido & Materiales & Finalidad & Tiempo \\
\hline $\mathrm{R}$ & $\begin{array}{l}\text { Resumen de } \\
\text { la clase }\end{array}$ & $\begin{array}{l}\text { Se hace un resumen de la clase, } \\
\text { consolidando aquellos aspectos } \\
\text { más interesantes. }\end{array}$ & PPt & $\begin{array}{l}\text { Integrar el conocimiento } \\
\text { actual del alumno antes del } \\
\text { Role Play }\end{array}$ & $15^{\prime}$ \\
\hline D & Dudas & & PPt & & $10^{\prime}$ \\
\hline RP & Role Play & $\begin{array}{l}\text { Cada grupo de alumnos se } \\
\text { dividirán en grupos de } 4 \\
\text { personas. } \\
\text { "2 Alumnos harán las veces de } \\
\text { médico adjunto y residente. } \\
\text { "2 Alumnos harán las veces de } \\
\text { enfermo y acompañante. } \\
\text { Se realizará un guión por cada } \\
\text { g rupo co n e s ce n a ri o s } \\
\text { preestablecidos. } \\
\text { a) Paciente ingresado. } \\
\text { b) P a ci e n te tra s a I t a } \\
\text { hospitalaria. } \\
\text { c) Paciente ambulatorio. }\end{array}$ & $\begin{array}{c}\text { Mesa y silla } \\
\text { de } \\
\text { despacho, } 1 \\
\text { camilla, } 2 \\
\text { sillas de } \\
\text { acompañant } \\
\text { e. } \\
\text { Material } \\
\text { médico } \\
\text { (cinta } \\
\text { métrica, } \\
\text { cronómetro, } \\
\text { goniómetro, } \\
\text {...) }\end{array}$ & $\begin{array}{l}\text { Que el propio alumno } \\
\text { experimente las distintas } \\
\text { perspectivas que pueden } \\
\text { desarrollarse durante una } \\
\text { consulta médica. }\end{array}$ & $60^{\prime}$ \\
\hline
\end{tabular}

Ciclos de Mejora en el Aula (2020). Experiencias de Innovación Docente de la US Esta obra se distribuye con la licencia Creative Commons 


\section{Cuestionario inicial-final}

Como docentes pretendemos que nuestros/as alumnos/as tengan un pensamiento más reflexivo, abierto, autónomo y creativo. Darles las soluciones a las dudas y problemas que les plantean las materias y otras situaciones, sabemos que no les ayuda a ello, aunque sin ser conscientes a veces tendemos a darlas. Ayudarles a pararse a pensar y reflexionar sobre lo que está pasando en una situación específica y compleja no es perder el tiempo (Bain, 2005). Les proporciona nuevas perspectivas para resolver los conflictos.

Hay cuatro principios que definen a un modelo de enseñanza alternativo a lo tradicional: tomar en consideración los modelos mentales de los estudiantes, formular los contenidos como preguntas claves, utilizar una metodología basada en la investigación y concebir la evaluación con un proceso que retroalimente el aprendizaje (Porlán, 2017).

En este sentido vertebrar un cuestionario inicial y final para valorar la progresión que el grupo y cada alumno ha experimentado durante el desarrollo de la clase. Es una forma eficaz para que el alumno valore su progresión. También nos permite conocer una progresión del grupo y por ende, conocer nuestro impacto en el mismo. En este sentido se desarrollaron 4 preguntas que fueron valoradas al principio y al final de la clase.

1. ¿Cómo definirías la Sarcopenia?

2. ¿Qué estructura debe tener un texto científico que desea ser publicado?

3. Localiza con un círculo, ¿cuál desearías que fuera la localización que debe ocupar un biomédico en una consulta médica?

Ciclos de Mejora en el Aula (2020). Experiencias de Innovación Docente de la US Esta obra se distribuye con la licencia Creative Commons 


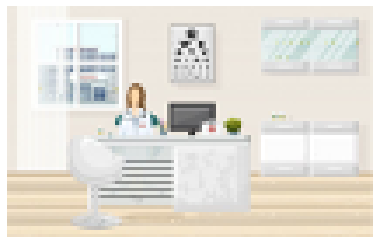

4. Localiza con un círculo, ¿cuál es la localización que actualmente ocupa un biomédico en una consulta médica?



\section{Aplicación del CIMA}

\section{Resumen de las sesiones}

Se trata de un grupo reducido de alumnos, de 2 o curso del grado de Biomedicina. Es una clase "práctica" que intenta suplir el déficit que ha ocasionado la pandemia por la COVID-19, dejando fuera de las consultas a estos estudiantes.

Mi principal motivación a la hora de diseñar la clase era clara: acercar a los estudiantes el ambiente de una consulta, dejar claro cuál debía de ser su papel en el transcurso de la misma y que conocieran la estructura "intelectual" sobre la que se desarrolla la entrevista médico-paciente.

Una consulta médica es tan "atractiva y sugerente" que reconocía de inicio que nada la suplantaría en la misma medida. Pero tenía que acercarme a ella. En esta ocasión,

Ciclos de Mejora en el Aula (2020). Experiencias de Innovación Docente de la US Esta obra se distribuye con la licencia Creative Commons 
comencé la clase con una presentación del alumnado, pero accedí a las fichas de los mismos, de modo que conocía algunos datos que hicieron que la presentación fuera más dinámica y amena (lugar de procedencia, domicilio actual, foto antes de entrar en la Facultad,...). Esto gustó bastante y ocasionó que "rompiéramos el hielo" de una manera rápida y natural. Si había cambios físicos notables en estas fotos, pedía permiso para proyectar la foto y ver las diferencias y esto ocasionó momentos muy divertidos.

Mi CIMA se inicia unas $72 \mathrm{~h}$ antes, cuando reciben por correo un podcast de unos $15 \mathrm{~min}$, en el que les explico el concepto de Sarcopenia. Decidí que fuera este concepto, ya que la mayoría de ellos lo desconocerían, pero resulta ser un concepto muy común y frecuente y, muy actual dadas las condiciones que ha generado y genera esta pandemia. El concepto de pérdida de masa muscular secundario a hospitalizaciones prolongadas y/o confinamiento generó en los estudiantes un doble impacto: expectación y conexión con el medio. Esto lo comprobé con el wooclap. A través de una nube de ideas les dije que expresaran no sólo términos asociados al concepto de sarcopenia, sino también las sensaciones que habían tenido durante la escucha del podcast.

Contextualizar el concepto de sarcopenia en el momento actual fue distendido y la mayoría de los alumnos intervinieron dando su opinión. No obstante, cuando les hice una pregunta para que buscaran un sentido más profundo de su exposición inicial, les encontré más incomodos. No obstante, reconozco que las "mascarillas" me lastraron a la hora de ver en gran medida las emociones del alumnado.

En este nivel de la clase, los vi confiados y tuve la sensación de que estaban disfrutando, pero aún así seguía detectando un eje muy acusado de profesor-alumno; me 
gustaría que hubiera habido más retroalimentación alumno-profesor. Es en este momento, antes de comenzar con la lectura crítica del artículo científico, cuando sin estar previsto en el "guión" de la clase, decidí dar una visión personal de las posibles salidas profesionales a las que pueden optar al término de sus estudios. Este hecho les impactó mucho. Sirvió como debate profundo, que si bien estaba fuera de la intención de la clase (el tema de la clase) me acercó a ellos. No sólo como elemento docente sino como probable "mentor" en un futuro. De hecho, he recibí numerosos emails en este sentido.

Antes de realizar una lectura crítica del artículo, les ayudé a comprender cuáles eran los aspectos más relevantes en este punto de la clase. Conocer la estructura de un documento científico, su rigurosidad, las fórmulas de expresión formales, ... Reconozco que los veía con cierto temor porque tenían un reto, que de forma individual tenían que solventar. Además, era un artículo que habíamos escrito desde mi servicio del H. U. Virgen del Rocío y eso podía generarle aún más dudas. Tras analizar el artículo, cada uno de ellos realizó un análisis crítico no sólo sobre la estructura del texto, sino de su contenido. Este aspecto les gustó, ya que probablemente uno de los caminos que deban desarrollar en un futuro laboral cercano, sea la realización de textos científicos.

Tras esto, comenzamos con agrupar a la clase tras la realización del "role play". Para mí, era el punto más determinante en el desarrollo de la clase. Era una forma de desarrollar el contenido de una manera natural. Les encantó la idea de tener que reproducir una consulta médica. Les indiqué incluso que fueran a buscar todo lo necesario a lo largo de las consultas del hospital. Tuvieron que salir por una silla de ruedas, una bata y un fonendoscopio. Esto generó mucho dinamismo y provocó que se incluyeran en el papel que cada uno iba a desarrollar. Les indiqué 
la necesidad de "guionizar" sus actuaciones. Que lo escribieran y tras esto lo analizaran. Agradecieron este mensaje y obtuve una respuesta positiva durante la ejecución del "role". Al final de cada una de las actuaciones, analizamos las fotografías que había tomado desde arriba, para valorar los flujos que se habían desarrollado durante la consulta médica. Obtuvieron una respuesta gráfica, del posicionamiento actual del biomédico en una estructura sanitaria y fuimos críticos sobre este concepto. Ellos comprendieron que tenían responsabilidad sobre su papel en el mundo sanitario, ya que son una Facultad de corto desarrollo y, por ende, con necesidades actuales de ubicación. Creo que analizaron que tenían un cometido para con sus intereses, más allá del académico.

Estoy satisfecho con mi labor como docente en el desarrollo de mi CIMA. El contenido del mismo y la intención sacaron al alumno del mero hecho académico. Proyectamos líneas futuras de actuación y, sobre todo, hablamos del futuro profesional de cada uno de ellos y también de la Biomedicina actual. Tengo la sensación de haberles llenado ambos bolsillos. El académico, ya que vía el concepto de Sarcopenia, han desarrollado habilidades necesarias para su desarrollo profesional; y el de su futuro laboral. Este último va más allá del simple hecho de conseguir trabajo. No es ese el motivo. Ser críticos con su futura profesión y conocer la posibilidad de transformarla hacia sus intereses actuales. Esto último es quizás lo más importante. Han aprendido que los caminos son diversos y que, en esa diversidad, sólo la profesionalidad y la búsqueda constante de innovación y originalidad, les hará alcanzar sus metas.

En relación a las debilidades, me centro en la mayor de ellas. Una práctica médica, no podrá ser nunca sustituida por otra fórmula. Falta el paciente y su problema. Lo puedes imitar, pero nunca será igual. Hay que asumir esta 
situación y partir de esta base. Quizás tendría que haber trabajado en este concepto. No lo sé. Pero es la sensación que he alcanzado tras el desarrollo de mi CIMA.

La enseñanza colegiada (dar clases con un colega) muy recomendada en muchos modelos me resulta atractiva (Finkel, 2008). La posibilidad de sentir que ellos están presenciando la experiencia intelectual de otros en lugar de tener la suya propia, puede generar en los estudiantes y en nosotros los docentes un efecto plural. Me siento satisfecho. Ahora más que nunca. Me gusta dar clases y me divierte, pero ahora tengo más recursos. Soy más elástico durante el desarrollo de las mismas.

\section{Evaluación del aprendizaje de los estudiantes}

Si preguntamos a un estudiante qué significa la evaluación del aprendizaje, probablemente nos dirá: "exámenes", y si le preguntamos a un profesor podría contestarnos: Es algo dificil que toma tiempo y experiencia, por lo que generalmente no me pagan, y para lo que no fui capacitado (Boursicot 2012). Pensamos que la mayor parte de lo que enseñamos es aprendido por los estudiantes, aunque la única manera de conocer los efectos de la enseñanza es realizar una evaluación continua y técnicamente adecuada, alineada con los planes de estudio de cada universidad o centro educativo y métodos de enseñanza. Estos métodos han de incluir al estudiante como entidad activa en el proceso.

Existen varias definiciones de evaluación, una de las más utilizadas es: Término genérico que incluye un rango de procedimientos para adquirir información sobre el aprendizaje del estudiante, y la formación de juicios de valor respecto al proceso de aprendizaje (Miller, 2012). 
Evaluación implica obtener información de diferentes fuentes como realimentación, exámenes, tareas y diversas interacciones con el educando. Los profesores que interactuamos con estudiantes debemos incorporarla desde una visión más profunda, como sugirió Derek Rowntree (1977): cuando una persona, con algún tipo de interacción directa o indirecta con otra, obtiene e interpreta información de manera consciente sobre el conocimiento y la comprensión, habilidades y actitudes de la otra persona. Hasta cierto punto evaluación es un intento de conocer a esa persona.

En este sentido generar un cuestionario de preguntas claves y a su vez valorar la progresión individual y colectiva de forma escalonada, es un modelo sólido a la hora de evaluar a nuestros estudiantes. A continuación, se expresa una tabla de niveles por preguntas (tabla 4), tabla por niveles por preguntas según cada alumno (tabla 5) y finalmente las 4 escaleras de aprendizaje (figuras 3-6).

Tabla 4. Tabla de niveles por preguntas. E1 (Escalón 1); E2 (Escalón 2); E3 (Escalón 3)

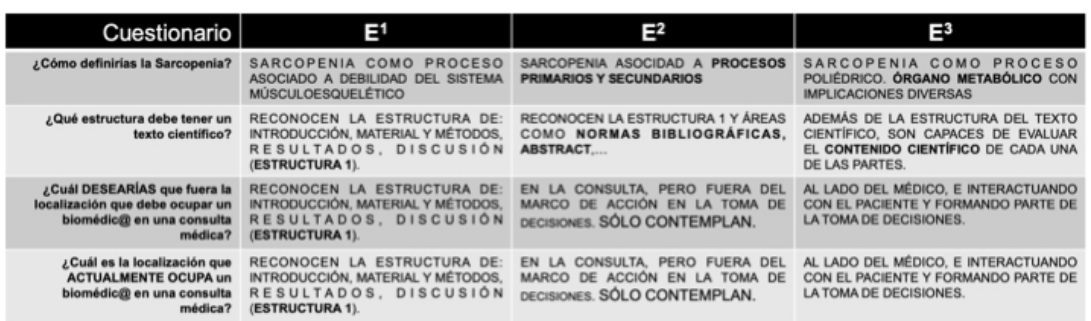

Ciclos de Mejora en el Aula (2020). Experiencias de Innovación Docente de la US Esta obra se distribuye con la licencia Creative Commons 
Tabla 5. Tabla de niveles por preguntas según cada alumno de la clase. E1 (Escalón 1); E2 (Escalón 2); E3 (Escalón 3). A1-6 (Alumno 1-6). Cuestionario 1-4, corresponde a las preguntas número 1-4 del cuestionario

\section{Progresión de Agrundizajo del alumna.}

\begin{tabular}{|c|c|c|c|c|c|c|c|c|c|}
\hline & \multicolumn{2}{|c|}{$\begin{array}{c}\text { CUESTIONARIO } 1 \\
\text { Ondide:lins }\end{array}$} & \multicolumn{2}{|c|}{$\begin{array}{c}\text { CUESTIONASEIO } 2 \\
\text { |nichami }\end{array}$} & \multicolumn{2}{|c|}{$\begin{array}{c}\text { CUESTIONARIO } 3 \\
\text { Onidalens) }\end{array}$} & \multicolumn{2}{|c|}{$\begin{array}{c}\text { CUESTIONAAIO } 4 \\
\text { GrikOAmine }\end{array}$} & \multirow{2}{*}{$\frac{\text { suma }}{+5}$} \\
\hline $\mathbf{A}^{\mathbf{1}}$ & E1 & E2 & 62 & $E 2$ & $\mathbf{E 1}$ & $\mathrm{E3}$ & E1 & $\mathrm{E}$ & \\
\hline$A^{2}$ & Ea & 12 & t1 & 12 & e1 & E3 & $\mathbf{n}$ & E & +6 \\
\hline$A^{\prime}$ & E1 & E2 & E1 & $E 2$ & E1 & $E 2$ & E1 & $E 2$ & +4 \\
\hline $\mathbf{A}^{4}$ & $\mathbf{E}$ & E3 & 61 & 12 & 62 & 63 & $\mathbf{B}$ & 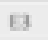 & +5 \\
\hline$A^{5}$ & $\varepsilon 2$ & E2 & E1 & $E 3$ & E2 & $E 2$ & E2 & $E 2$ & +2 \\
\hline$A^{*}$ & EI & E3 & E1 & E3 & E3 & $\mathrm{E} 3$ & $E_{3}$ & $E_{3}$ & +4 \\
\hline
\end{tabular}

En la primera escalera se observa la progresión que el alumno desarrolla, cuando se le solicita una definición de sarcopenia. El concepto de sarcopenia en el que se evalúa al músculo no sólo como componente del sistema músculo-esquelético, sino como órgano metabólico, es la mejor definición posible para este término.

Cuestionario 1 | Cómo definirias Sarcopenia



Figura 3. Escalera de aprendizaje de la pregunta 1 del cuestionario: ¿Cómo definirías la sarcopenia?

El concepto de sarcopenia se transforma en una "excusa" para definir cuál es la estructura de un texto Ciclos de Mejora en el Aula (2020). Experiencias de Innovación Docente de la US 
científico. Los biomédicos deben alcanzar un gran dominio en la realización (forma y contenido) de estos textos. Tener la capacidad de evaluar la estructura del documento, pero, sobre todo, el contenido del mismo se erige como el punto clave para alcanzar la mayor puntuación posible en este apartado.

\section{Cuestionario 2 | Estructura de un texto científico.}

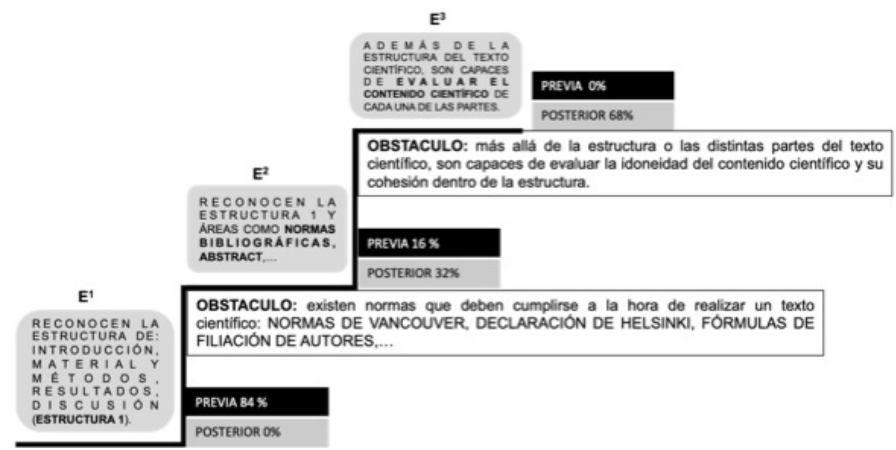

Figura 4. Escalera de aprendizaje de la pregunta 2 del cuestionario: Estructura de un texto científico

El papel que ocupa o que debería ocupar el biomédico en una consulta médica, protagonizan los cuestionarios 3 y 4. Es una profesión que aún está en vías de evolución. Probablemente, estos nuevos biomédicos definan las "líneas maestras" de esta nueva profesión. La necesidad de unirse a la práctica clínica habitual les hará más realistas en sus proyecciones científicas. Que la investigación básica tenga su repercusión en la práctica clínica habitual, puede venir de la mano de la integración del biomédico en la consulta médica.

Ciclos de Mejora en el Aula (2020). Experiencias de Innovación Docente de la US Esta obra se distribuye con la licencia Creative Commons 


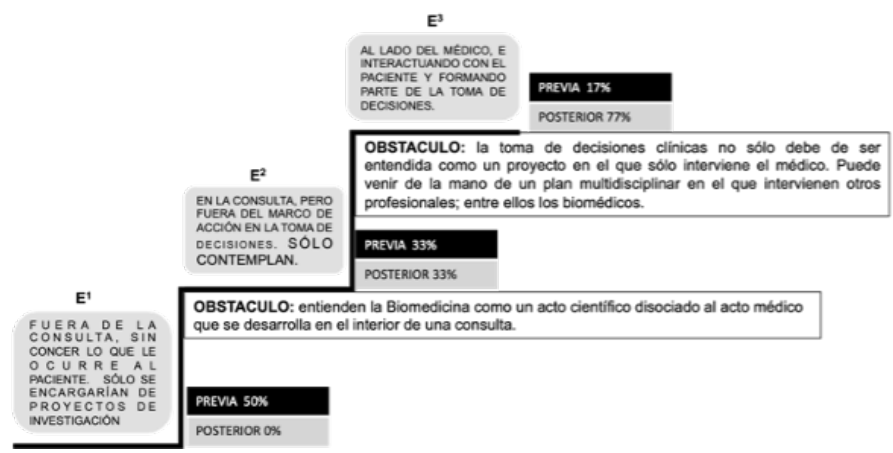

Figura 5. Escalera de aprendizaje de la pregunta 3 del cuestionario: ¿Cuál desearías que fuera la localización que debe ocupar un biomédico en una consulta médica?



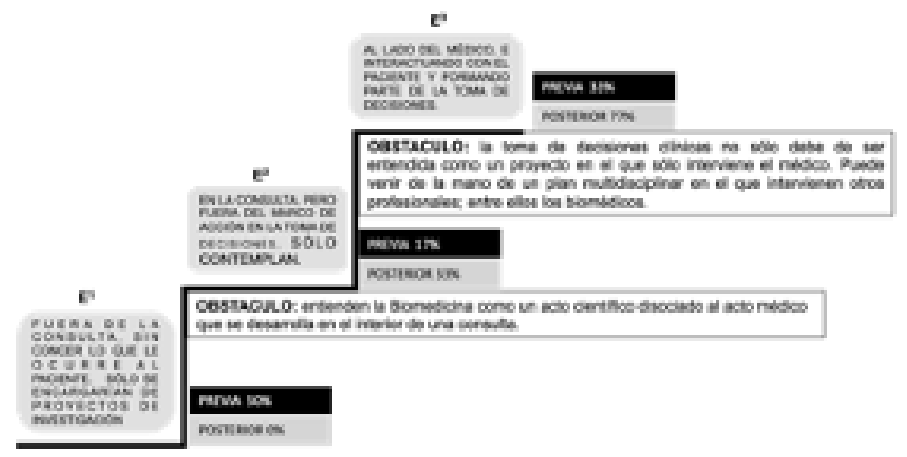

Figura 6. Escalera de aprendizaje de la pregunta 4 del cuestionario: ¿Cuál es la localización que actualmente ocupa un biomédico en una consulta médica?

\section{Evaluación del CIMA}

\section{Cuestiones a mantener y cambios a introducir para un futuro Ciclo}

Mi CIMA intentaba salvar tres hitos que necesariamente precisaban de una adaptación progresiva. Cambiar tu modelo de impartir clases (reto personal), hacer cambiar el

Ciclos de Mejora en el Aula (2020). Experiencias de Innovación Docente de la US (c) Esta obra se distribuye con la licencia Creative Commons 
modelo de aprendizaje de los alumnos (reto en el alumnado) y sobrepasar aquellas barreras que la "tendencia o la tradición" han establecido dentro del modelo de clase en la Facultad de Medicina. Este modelo "academicista" viene impulsado probablemente por una sensación de seguridad colectiva del profesorado por dos ítems, que tienen mucho peso en el día a día de cualquier asignatura: a) El examen de la asignatura, que se realiza entre los 3 hospitales de la Facultad. b) El "famoso" examen MIR. Son dos situaciones que probablemente limite la maniobrabilidad de las clases. La falta de veteranía en una institución también debe de ser contemplada en este proceso.

Es por ello por lo que decidí realizar mi modelo docente en el Grado de Biomedicina. Es una Facultad "joven", con modelos aún por afianzar y sin esas limitaciones (MIR y examen final colectivo entre los exámenes finales) que asumo, me inquietaban dentro de la Facultad de Medicina. Este alumnado tiene necesidad de realizar un abordaje clínico en su aprendizaje, ya que tienen sólo esta oportunidad de cara a acercarse a un entorno eminentemente clínico. El modelo se desarrolló dentro de las clases prácticas del 2 o curso del grado de Biomedicina. Son grupos pequeños, de entre 5-7 estudiantes. La intención, no era otra que intentar reproducir lo que ocurre en una consulta médica y evaluar el papel que un biomédico desarrolla en la actualidad. Cuál será el futuro de ellos, -en el desempeño de sus funciones como clínicos adyacentes a la práctica clínica habitual- era quizás el fin último en el destino de mis clases.

Encontré un grupo muy disociado entre los distintos alumnos. Eran como entidades independientes que apenas interactuaban entre ellos y menos aún en el eje alumno-profesor. Se sentían más cómodos, aunque menos activos en el tradicional eje profesor-alumno. Había mucha timidez en el grupo, aunque tenía claro que tenía 
que detectar algún estudiante que me catalizara un dinamismo en el grupo. Que me sirviera como referente para extenderse dentro del grupo. De hecho, en la presentación, intentaba dos cosas: conocer el nivel inicial del grupo (ZDP) y conocer "su personalidad" dentro de la clase. Para ello, disponía de las fichas de cada alumno y según la información que dispongo (edad, procedencia, vivienda habitual, ...), intentaba sacar unas primeras conclusiones en este sentido.

Las sensaciones iban mejorando conforme se desarrollaba la clase, que tenía un destino final muy "acusado" como era el de desarrollar un modelo de consulta médica que imitara al real. Usar un concepto como la Sarcopenia, generaba un pilar para distribuir las fuerzas: a) Era un concepto novedoso, con matices clínicos y de investigación. b) Era una situación clínica con gran implicación actual en la Pandemia por la COVID-19. c) Por todo ello, me servía de puente entre el texto científico o la investigación y el interior de la consulta médica. No olvidemos, que era una clase práctica no una clase teórica o de conceptos.

El desarrollo individual de la clase era más bien lento e insisto el abordaje "intelectual" de las respuestas ante mis preguntas no generaba el debate deseado. No obstante, cuando llegó el momento de agruparlos, todo cambió. Desarrollar la clase a partir del planteamiento de un problema. Esto en Medicina y Biomedicina, se consigue de forma muy concreta a través del planteamiento de una clase gracias a los casos clínicos. Estos casos clínicos ponen en contexto al alumno y lo mantienen expectante durante la sesión. Es mucho más interesante presentarlo al principio y valorar las ideas iniciales de las que parten los alumnos (ZDP), pues de este modo, la enseñanza va a ir mucho más orientada a las necesidades que demandan los mismos.

Ciclos de Mejora en el Aula (2020). Experiencias de Innovación Docente de la US Esta obra se distribuye con la licencia Creative Commons 
Para un futuro CIMA pretendo introducir más sesiones de trabajo en equipo y reducir el trabajo individual. Considero que, al trabajar en grupo, los alumnos desarrollan mejor sus habilidades y se estimulan entre ellos.

La búsqueda activa de información y ver el efecto de cómo el alumno modifica su esquema tras la inclusión de un problema (actividad de contraste), se me antojan claves a la hora del diseño de un nuevo ciclo de mejora más amplio.

\section{Aspectos de la experiencia que se pretenden incorporar a toda la práctica}

Cuestionario de ideas iniciales y finales.

Comentar con los alumnos las principales ideas iniciales desde las que parte el conocimiento, formar grupos y volver a desarrollarlo, previo a la realización de actividades de contraste, que sirvan como fuente de inspiración orientada.

También me resulta de gran utilidad, el cuestionario de expectativas previas del alumno y el cuestionario de satisfacción posterior. De este modo, como docente valoras las necesidades de los alumnos y los aspectos a mejorar en futuras sesiones.

La enseñanza colegiada (dar clases con un colega).

\section{Principios Didácticos argumentados}

Desarrollar un "role play" sobre el tema en cuestión, generó un cambio sustancial en el desarrollo de la clase. Recuerdo, perfectamente que guionizar esta tarea, la necesidad de escribir lo que ellos pensaban dejarlo plasmado

Ciclos de Mejora en el Aula (2020). Experiencias de Innovación Docente de la US Esta obra se distribuye con la licencia Creative Commons 
en papel, les ayudó mucho. De hecho, he de reconocer que esa reflexión más amplia de escribir las ideas ha sido uno de los hitos más importantes en el desarrollo de las clases, tanto para los alumnos como para el mío propio. El análisis intelectual se hace más acusado cuando escribes lo que piensas. "Guionizar" la clase y estructurarla de forma física en un papel, ha sido de los mejores consejos que he recibido y que he podido trasladar a los alumnos.

Los tipos de contenidos gracias al uso de Mapa de Contenidos fue el mayor reto al que tuve que hacer frente. Desconocía su función y ejecución y gracias a las recomendaciones realizadas por Rafael Porlán y apoyándome en los modelos de otros compañeros, supe de su realización $y$, sobre todo, de su importancia de cara al desarrollo sólido de una clase.

Una cuestión que también la identifiqué como de gran valor para mí y para los alumnos, era generar una pregunta de contraste. En el "role play" me ayudó mucho cambiar sobre la marcha el contexto clínico que inicialmente se les había determinado. Delataba el grado de aprendizaje de cada alumno y me permitía dirimir el grado de integración de cada uno de ellos. Es otro de los bloques que destacaría más en este sentido.

En cuanto a la evaluación he de reconocer que el desarrollo de un diagnóstico inicial y final de las ideas, valorar los modelos mentales de los estudiantes, los obstáculos de aprendizaje y las escaleras de aprendizaje y evaluación han sido una gran sorpresa para mí. Desconocía de estos recursos y estoy convencido que son el camino para evaluar de forma "real" el potencial de cada uno de los estudiantes, más allá de la tradicional prueba de examen.

Ciclos de Mejora en el Aula (2020). Experiencias de Innovación Docente de la US Esta obra se distribuye con la licencia Creative Commons 
Palabras clave: Grado de Medicina, Docencia Universitaria, Experimentación Docente Universitaria, Sarcopenia.

Keywords: Medical Degrre, University Teaching, Universitiy Teachin Experimentation, Sarcopenia.

\section{Referencias bibliográficas}

Bain, K. (2005). Lo que hacen los mejores profesores universitarios. Valencia: Publicaciones Universidad de Valencia.

Boursicot, K., Etheridge, L., Setna, Z., Sturrock, A., Ker, J., Smee, S. y Sambandam, E. (2011). Performance in assessment: consensus statement and recommendations from the Ottawa conference. Med Teach, 33(5), 370-83.

Derouesn, C. (2017). Lev Semenovich Vygotsky (1896-1924): an introduction to the historico-cultural psychology and the development of the higher psychological processes. Geriatr Psychol Neuropsychiatr Vieil, 15(3), 295-307.

Finkel, D. (2008). Dar clases con la boca cerrada. Valencia: Publicaciones Universidad de Valencia.

Miller, M. D., Linn, R. L. y Gronlund, N. E. (2012). Measurement and Assessment in Teaching. USA: Pearson.

Porlán R. (2017). Enseñanza universitaria. Cómo mejorarla. Madrid: Morata

Rowntree, D. (1977). Assessing students: How shall we know them? London: Kogan Page.

Ciclos de Mejora en el Aula (2020). Experiencias de Innovación Docente de la US Esta obra se distribuye con la licencia Creative Commons 